\title{
Modal Characteristics of ARROW-Type Vertical-Cavity Surface-Emitting Lasers
}

\author{
Tae-Woo Lee, Student Member, IEEE, Susan C. Hagness, Member, IEEE, Delai Zhou, Student Member, IEEE, \\ and Luke J. Mawst, Senior Member, IEEE
}

\begin{abstract}
Antiresonant reflecting optical waveguide (ARROW-type) vertical-cavity surface-emitting lasers are designed for high-power single-mode operation. A detailed full-vector finite-difference time-domain (FDTD) study shows strong modal discrimination in favor of the fundamental mode for large aperture $(>6 \mu \mathrm{m})$, large index-step $(\Delta n>0.025)$ simplified ARROW-type devices. The FDTD model identifies the polarization-dependent radiation losses of the higher order modes, which prevent them from reaching laser threshold.
\end{abstract}

Index Terms-Full-vector finite-difference time-domain methods, semiconductor laser, vertical-cavity surface-emitting laser.

\section{INTRODUCTION}

$\mathbf{S}$ TABLE single-transverse-mode vertical-cavity surface-emitting lasers (VCSELs) with output powers greater than $10 \mathrm{~mW}$ have not yet been realized. One of the major limitations to achieving reliable high-power single-mode VCSELs is the difficulty in fabricating larger aperture single-mode devices, due to poor intermodal discrimination, gain-spatial-hole-burning (GSHB), and thermal lensing [1]. Large-aperture devices exhibit lower overall electrical resistance and thermal impedance, and result in higher output power operation because of reduced heating. In addition, a lower operating current density could lead to reliable operation at high output powers.

Most of the previous approaches to achieving single-mode operation have focused on the use of a built-in positive-index waveguide [2]. For such a structure, the cutoff aperture is limited by the device index-step, requiring a relatively small index step to insure single-mode operation. More recently, larger aperture (diameters greater than $6 \mu \mathrm{m}$ ) positive-index-guided VCSELs have been realized by way of preferential pumping of the fundamental mode [3], modifying the VCSEL surface [4], or extending the laser cavity [5], with record-high 5.5-mW single-mode output power achieved [5].

Alternatively, the use of a negative index guide (antiguide) eliminates the cutoff requirement, since high-order modes are suppressed by strong mode-dependent lateral radiation losses [6]. Because of the strong lateral edge-radiation losses for higher order modes, antiguided structures can remain single mode to many times the threshold current, even from large aperture devices with large lateral index step. The large built-in index step

\footnotetext{
Manuscript received April 4, 2001. This work was supported by the National Science Foundation Presidential Early Career Award for Scientists and Engineers ECS-9985004, and by the National Science Foundation Faculty Early Career Development Award ECS-9734283.

The authors are with the Department of Electrical and Computer Engineering, University of Wisconsin-Madison, Madison, WI 53706 USA.

Publisher Item Identifier S 1041-1135(01)06436-9.
}

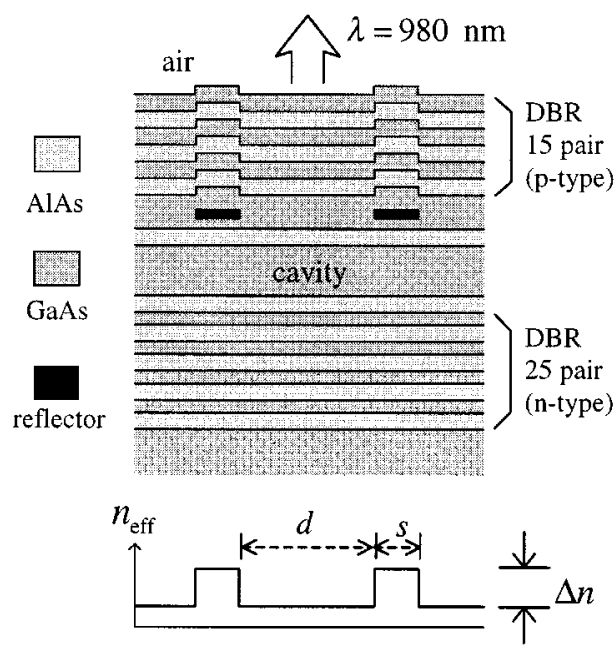

Fig. 1. Schematic diagram showing a cross-sectional cut through the cylindrical S-ARROW VCSEL structure.

$(\Delta n>0.025)$ provides for mode stability against carrier and thermally induced index variations above threshold. Antiguided VCSELs have been fabricated either by surrounding a low-index core region with a high-index material via a regrowth process [7], [9], or by shifting the cavity resonance toward a longer wavelength outside the core to create an effective low-index core region [8]. The latter structure relies on the cavity-induced index step proposed by Hadley [10]. Although these devices display promising results, such as single-mode operation up to 5-15 $\times I_{\text {th }}$ for diameters as large as $16 \mu \mathrm{m}$, the power has been limited to less than $2 \mathrm{~mW}$ because of the relatively large radiation loss incurred for the fundamental mode, which is inherent to the antiguide structure.

A novel simplified antiresonant reflecting optical waveguide (S-ARROW)-type structure- a direct modification of the antiguide-offers an effective means of lowering the radiation losses for the fundamental mode while maintaining strong mode discrimination [11]. In this letter, we present a comprehensive analysis of the cold-cavity modal characteristics of large-aperture S-ARROW VCSELs using full-vector finite-difference time-domain (FDTD) simulations [12]. Strong suppression of higher order modes is predicted from large aperture $(6-8 \mu \mathrm{m}$ in diameter) large index-step $(\Delta n>0.025)$ structures.

\section{S-ARROW VCSEL DESIGN}

In the S-ARROW structure of Fig. 1, a low-index core region is surrounded by lateral-quarter-wave reflector regions, which are designed to be antiresonant for the lateral radiation of the 
fundamental mode. This design minimizes the loss of the fundamental mode. Since higher order modes do not meet the antiresonant condition, their losses remain large, leading to a highthreshold gain requirement for those modes. Single-mode operation has been reported from S-ARROW VCSELs [13] with diameters as large as $12 \mu \mathrm{m}$, although output powers were limited by thermal saturation to $1 \mathrm{~mW}$. Optimization of the growth and fabrication process is expected to allow single-mode output powers in the 5-10-mW range for devices with 6-8 $\mu$ m-diameter apertures.

The lateral waveguide for the S-ARROW structure is created by locally shifting the cavity resonance toward longer wavelength in a ring surrounding the emitting aperture. Hadley [10] has shown that an increase in the resonant wavelength is equivalent to an increase of effective index according to the approximate relation: $\Delta n / n=\Delta \lambda / \lambda$. For a given $\Delta n$, a one-dimensional (1-D) model can be used to estimate the width of the reflecting ring $s$ that will satisfy the antiresonance condition, $s=m \lambda_{l} / 4$, where $m$ is an odd integer and $\lambda_{l}$ is the lateral wavelength in the high-index region [13]. For the index steps used here $(\Delta n=0.025$ and $\Delta n=0.05)$, we calculate $\lambda_{l}=2.4 \mu \mathrm{m}$ and $\lambda_{l}=1.7 \mu \mathrm{m}$.

\section{FDTD ModEL AND SimUlation RESUlTS}

Full-vector FDTD solutions of Maxwell's equations in cylindrical coordinates [12] are useful for investigating transverse mode selectivity in complex VCSEL structures, as demonstrated previously for air-post index-guided VCSELs [14]. Since the S-ARROW VCSEL exhibits rotational symmetry, the azimuthal $(\phi)$ dependence of the vector field components can be expressed as a Fourier series and accounted for analytically, eliminating the need for gridding in the $\phi$-direction. The FDTD simulation is simply rerun for each azimuthal mode of interest. In the FDTD computational domain, the following material properties were assumed: $n=3.5$ and 2.95 for the refractive index of GaAs and AlAs, respectively, and $\sigma=0.1115$, $0.2137,0.0940$, and $0.1801 \mathrm{~S} / \mathrm{cm}$ for the conductivity of $\mathrm{n}$ : GaAs, p:GaAs, n:AlAs, and p:AlAs, respectively. The computational domain was terminated with optimized uniaxial perfectly matched layer absorbing boundary conditions.

The key modal characteristic is the quality factor $(Q)$, which is inversely proportional to the mode-dependent lateral radiation losses. The resonant frequency and $Q$ of each transverse mode was obtained via spectral analysis of the time-domain impulse response of the cold-cavity VCSEL structure. To significantly reduce the simulation time required to accurately extract resonant frequencies and $Q \mathrm{~s}$, we processed the early-time response using a Pade/FFT linear extrapolation scheme [12]. The modal identity of the cavity resonance was obtained from knowledge of the azimuthal mode number assumed in the simulation and from observations of the transverse modal profile for a single-frequency source excitation at the resonant frequency.

We conducted a comprehensive FDTD analysis of the modal characteristics of the VCSEL structure shown in Fig. 1 as a function of the following design parameters: 1) width of reflector $s ; 2)$ cavity diameter $d$ defined as the diameter of the inner rim of the reflector ring; and 3) radial effective index step, $\Delta n$
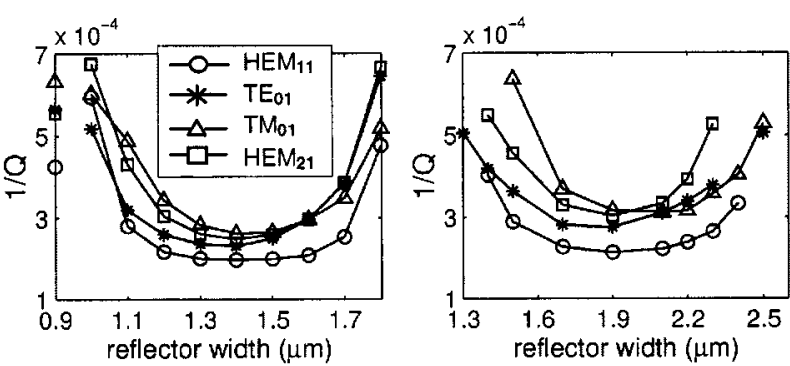

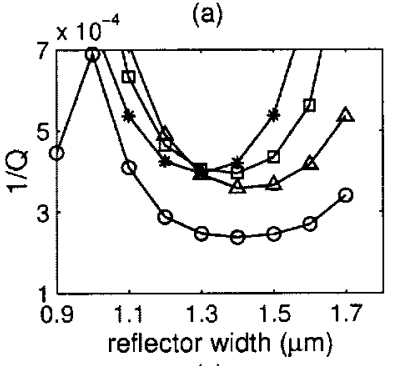

(c)

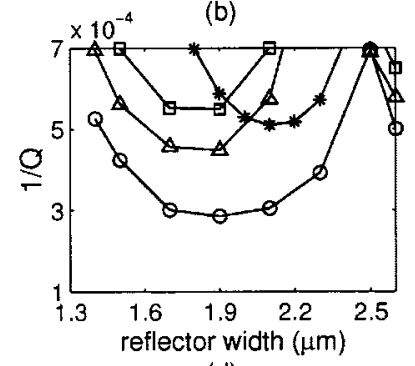

(d)
Fig. 2. FDTD-computed inverse of the quality factor as a function of reflector width for the following cavity diameters and index steps: (a) $d=8 \mu \mathrm{m}, \Delta n=$ 0.05 , (b) $d=8 \mu \mathrm{m}, \Delta n=0.025$, (c) $d=6 \mu \mathrm{m}, \Delta n=0.05$, and (d) $d=6 \mu \mathrm{m}, \Delta n=0.025$.

determined by the thickness and index of refraction of the reflector region. Results of this parametric study are presented in Fig. 2. The parameter space covers a range of reflector widths $(0.5 \leq s \leq 2.6 \mu \mathrm{m})$ for which the S-ARROW structure exhibits at least one antiresonance. Each graph in Fig. 2 shows the FDTD-computed loss curves $(1 / Q)$ of the four lowest order modes as a function of reflector width for fixed radial effective index steps $(\Delta n=0.05, \Delta n=0.025)$ and cavity diameters $(d=6 \mu \mathrm{m}, d=8 \mu \mathrm{m})$.

For the larger index step $(\Delta n=0.05)$, the $\mathrm{HEM}_{11}$ mode (fundamental mode) experiences the lowest radiation losses for a reflector width of $1.4 \mu \mathrm{m}$, irrespective of the cavity diameter. This optimum width increases to $1.9 \mu \mathrm{m}$ for the smaller index step $(\Delta n=0.025)$. At these reflector widths, the next higher order competing mode is the $\mathrm{TE}_{01}$ mode in the larger diameter cavities [see Fig. 2(a) and (b)], and the $\mathrm{TM}_{01}$ mode in the smaller diameter cavities [see Fig. 2(c) and (d)]. As the reflector width increases [or, in the case of Fig. 2(c), decreases] from those widths which yield the minimum loss, the polarization of the next higher order competing mode changes to the dual nonhybrid $(m=0)$ mode. The optimum reflector widths identified in our FDTD simulations are in good agreement with those values obtained using a 1-D scalar effective-index model. However, the polarization dependence of the lateral radiation losses are only understood through the full-vector FDTD simulations.

The optimum cavity design should offer not only small radiation losses $(1 / Q)$, but also strong mode discrimination. In each graph of Fig. 2, the losses of the higher order modes are substantially higher than those of the fundamental mode. To enable straightforward comparisons of mode discrimination capabilities of VCSEL structures with different design parameters, we calculated the difference $\Delta Q$ in quality factors of the fundamental and the next higher order competing mode. The mode discrimination characteristics for cavities designed with 
TABLE I

\begin{tabular}{c|c|c|c}
\hline$d$ & $\Delta n$ & Maximum $Q_{0}$ (non-optimum $\left.\Delta Q\right)$ & Maximum $\Delta Q$ (non-optimum $\left.Q_{0}\right)$ \\
\hline $8 \mu \mathrm{m}$ & 0.05 & $Q_{0} \cong 5070, \Delta Q \cong 750(s=1.4 \mu \mathrm{m})$ & $Q_{0} \cong 4800, \Delta Q \cong 1420(s=1.6 \mu \mathrm{m})$ \\
\hline $8 \mu \mathrm{m}$ & 0.025 & $Q_{0} \cong 4680, \Delta Q \cong 1050(s=1.9 \mu \mathrm{m})$ & $Q_{0} \cong 4500, \Delta Q \cong 1280(s=2.1 \mu \mathrm{m})$ \\
\hline $6 \mu \mathrm{m}$ & 0.05 & $Q_{0} \cong 4210, \Delta Q \cong 1420(s=1.4 \mu \mathrm{m})$ & $Q_{0} \cong 4050, \Delta Q \cong 1500(s=1.3 \mu \mathrm{m})$ \\
\hline $6 \mu \mathrm{m}$ & 0.025 & $Q_{0} \cong 3500, \Delta Q \cong 1270(s=1.9 \mu \mathrm{m})$ & $Q_{0} \cong 3280, \Delta Q \cong 1320(s=2.1 \mu \mathrm{m})$ \\
\hline
\end{tabular}

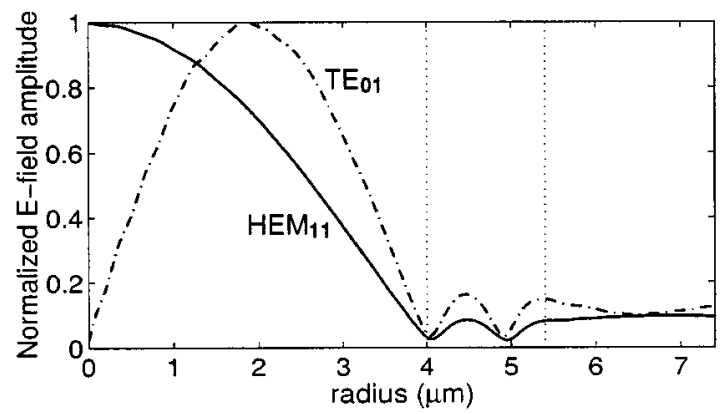

Fig. 3. FDTD-computed electric-field distributions as a function of radial distance for the two lowest order modes in an S-ARROW VCSEL cavity with $d=8 \mu \mathrm{m}, \Delta n=0.05$, and $s=1.4 \mu \mathrm{m}$.

reflector widths that yield the maximum quality factor for fundamental mode $\left(Q_{0}\right)$ are summarized in Table I.

The relatively flat nature of the loss curves for the fundamental mode can be exploited to achieve single-mode operation over a large range of reflector widths without a significant increase in loss in the fundamental mode. For example, $\Delta Q$ can be further increased from that achieved for the reflector widths identified in the first column of results in Table I, by simple increasing [or in the case of Fig. 2(c), decreasing] the reflector width slightly and accepting a slightly higher amount of radiation loss in the fundamental mode. The second column of results in Table I illustrates this point.

We excited the larger diameter larger index-step VCSEL cavity with a source tuned to the cavity's resonant frequency to visualize the electric-field profile along a transverse cut through the structure. The radial electric-field profiles of the two competing modes $\left(\mathrm{HEM}_{11}\right.$ and $\left.\mathrm{TE}_{01}\right)$ for $d=8 \mu \mathrm{m}, \Delta n=0.05$, and $s=1.4 \mu \mathrm{m}$ are shown in Fig. 3. The field variation inside the reflector region (bounded by the vertical dashed lines) illustrates the antiresonance condition $\left(s=3 \lambda_{l} / 4\right)$.

Finally, to illustrate the strong mode selectivity of the antiguided structure, we compared the mode selectivity of the S-ARROW VCSEL with that of an oxide-aperture VCSEL of similar material parameters. The design parameters we chose in our simulation of the oxide-aperture VCSEL $(d=8 \mu \mathrm{m}$, $\Delta n=0.025)$ are larger than conventionally used in these structures, but they enable a direct comparison with the large-aperture large-index-step S-ARROW VCSEL results of Fig. 2(b). Our FDTD-computed quality factors for the oxide-aperture structure are as follows: $Q=5920$ for the $\mathrm{HEM}_{11}$ mode and $Q=5820$ for the $\mathrm{TE}_{01}$ mode. Therefore, $\Delta Q \cong 100$ for the large-diameter large-index-step oxide-aperture VCSEL. In comparing with the results of Table I, we see that while the $Q$ of the fundamental mode in the oxide-aperture VCSEL is approximately $25 \%$ higher than the $Q$ of the funda- mental mode in the S-ARROW VCSEL, the mode selectivity of the oxide-aperture VCSEL, as measured by $\Delta Q$, is much poorer.

\section{CONCLUSION}

Our cold-cavity FDTD simulations have illustrated two important modal characteristics of the S-ARROW VCSEL: 1) the fundamental mode exhibits low radiation losses resulting in a relatively high cavity $Q$ and 2) higher order modes exhibit radiation losses that are substantially greater than those of the fundamental mode. As a result, the S-ARROW VCSEL shows much promise for achieving high-power single-mode operation with large apertures and large built-in index steps. Future studies are needed to ascertain the influence of GSHB and thermal lensing on the antiguided-VCSEL modes above laser threshold.

\section{ACKNOWLEDGMENT}

Computing resources were provided in part by Cray, Inc.

\section{REFERENCES}

[1] G. C. Wilson, D. M. Kuchta, J. D. Walker, and J. S. Smith, "Spatial hole burning and self-focusing in vertical-cavity surface-emitting lasers," Appl. Phys. Lett., vol. 64, pp. 542-544, 1994.

[2] D. L. Huffaker, D. G. Deppe, K. Kumar, and T. J. Rogers, "Native-oxide defined ring contact for low threshold vertical-cavity lasers," Appl. Phys. Lett., vol. 64, pp. 97-99, 1994.

[3] K. D. Choquette, A. J. Fischer, K. M. Geib, G. R. Hadley, A. A. Allerman, and J. J. Hindi, "High single mode operation from hybrid ion implanted/selectively oxidized VCSELs," in IEEE 17th Int. Semiconductor Laser Conf., Monterey, CA, Sept. 2000.

[4] H. J. Unold, M. Kicherer, S. W. Z. Mahmoud, R. Jager, R. Michalzik, and K. J. Ebeling, "Spatially varied anti-resonant DBR design for large-area single-mode VCSELs," in IEEE 17th Int. Semiconductor Laser Conf., Monterey, CA, Sept. 2000.

[5] S. W. Z. Mahmoud, H. J. Unold, W. Schmid, R. Jäger, R. Michalzik, and K. J. Ebeling, "Analysis of longitudinal mode wave guiding in verticalcavity surface-emitting lasers with long monolithic cavity," Appl. Phys. Lett., vol. 78, pp. 586-588, 2001.

[6] D. Botez, L. J. Mawst, and G. Peterson, "Resonant leaky-wave coupling in linear arrays of antiguides," Electron. Lett., vol. 24, pp. 1328-1329, 1988

[7] Y. A. Wu, G. S. Li, R. F. Nabiev, K. D. Choquette, C. Caneau, and C. J. Chang-Hasnain, "Single-mode, passive antiguide vertical-cavity surface emitting laser,” J. Select. Topics Quantum Electron., vol. 1, pp. 629-637, June 1995.

[8] T. H. Oh, M. R. McDaniel, D. L. Huffaker, and D. G. Deppe, "Cavity-induced antiguiding in a selectively oxidized vertical-cavity surface-emitting laser," IEEE Photon. Technol. Lett., vol. 10, pp. 12-14, Jan. 1998.

[9] B. S. Yoo, H. Y. Chu, M. S. Park, and E. H. Lee, "Stable transverse mode emission in vertical-cavity surface-emitting lasers antiguided by amorphous GaAs layer," Electron. Lett., vol. 32, pp. 116-117, 1996.

[10] G. R. Hadley, "Effective index model for vertical-cavity surface-emitting lasers," Opt. Lett., vol. 20, pp. 1483-1485, 1995.

[11] I. V. Goltser, L. J. Mawst, and D. Botez, "Single-cladding antiresonant reflecting optical waveguide-type diode laser," Opt. Lett., vol. 20, pp. 2219-2221, 1995.

[12] A. Taflove and S. Hagness, Computational Electrodynamics: The Finite-Difference Time-Domain Method, 2nd ed. Norwood, MA: Artech House, 2000.

[13] D. Zhou and L. J. Mawst, "Simplified-antiresonant reflecting optical waveguide-type vertical-cavity surface-emitting lasers," Appl. Phys. Lett., vol. 76, pp. 1659-1661, 2000.

[14] G. Liu, J. F. Seurin, S. L. Chuang, D. I. Babic, S. W. Corzine, M. Tan, D. C. Barnes, and T. N. Tiouririne, "Mode selectivity study of verticalcavity surface-emitting lasers," Appl. Phys. Lett., vol. 73, no. 6, pp. 726-728, 1998. 